Research report

\title{
Effects of exercise on depressive behavior and striatal levels of norepinephrine, serotonin and their metabolites in sleep-deprived mice
}

\author{
Thiago Medeiros da Costa Daniele ${ }^{\mathrm{a}, \mathrm{b}, \mathrm{c}, 1}$, Pedro Felipe Carvalhedo de Bruin ${ }^{\mathrm{a}, \mathrm{b}, \mathrm{c}, 1}$, \\ Emiliano Ricardo Vasconcelos Rios ${ }^{\mathrm{c}, 1}$, Veralice Meireles Sales de Bruin ${ }^{\mathrm{a}, \mathrm{b}, \mathrm{c}, 1, *}$ \\ a Programa de Pós-Graduação em Ciências Médicas, Universidade Federal do Ceará, Fortaleza, Brazil \\ b Sleep and Biological Rhythms Laboratory, UFC, Brazil \\ c Universidade Federal do Ceará (UFC), Brazil
}

\section{A R T I C L E I N F O}

\section{Keywords:}

Exercise

Sleep deprivation

Depression

Serotonin

Norepinephrine

Striatum

\begin{abstract}
A B S T R A C T
Exercise is a promising adjunctive therapy for depressive behavior, sleep/wake abnormalities, cognition and motor dysfunction. Conversely, sleep deprivation impairs mood, cognition and functional performance. The objective of this study is to evaluate the effects of exercise on anxiety and depressive behavior and striatal levels of norepinephrine (NE), serotonin and its metabolites in mice submitted to $6 \mathrm{~h}$ of total sleep deprivation (6hTSD) and $72 \mathrm{~h}$ of Rapid Eye Movement (REM) sleep deprivation (72h-REMSD). Experimental groups were: (1) mice submitted to $6 \mathrm{~h}$-TSD by gentle handling; (2) mice submitted to 72h-REMSD by the flower pot method; (3) exercise (treadmill for 8 weeks); (4) exercise followed by 6h-TSD; (5) exercise followed by 72h-REMSD; (6) control (home cage). Behavioral tests included the Elevated Plus Maze and tail-suspension. NE, serotonin and its metabolites were determined in the striatum using high-performance liquid chromatography (HPLC). Sleep deprivation increased depressive behavior (time of immobilization in the tail-suspension test) and previous exercise hindered it. Sleep deprivation increased striatal NE and previous exercise reduced it. Exercise only was associated with higher levels of serotonin. Furthermore, exercise reduced serotonin turnover associated with sleep deprivation. In brief, previous exercise prevented depressive behavior and reduced striatal high NE levels and serotonin turnover. The present findings confirm the effects of exercise on behavior and neurochemical alterations associated with sleep deprivation. These findings provide new avenues for understanding the mechanisms of exercise.
\end{abstract}

\section{Introduction}

Sleep disorders are common and potentially inflict negative effects on conditions of health and disease $[1,2]$. Sleep is vital for the maintenance of interconnections and neurotransmitters that are essential for emotion and memory [3]. Previously, Xie et al. demonstrated that sleep deprivation impairs the removal of neurotoxic waste products affecting functional performance $[3,4]$. In view of this, it seems right to assume that sleep is essential for the brain. Experimental procedures of sleep deprivation offer the opportunity to evaluate cerebral changes and behavior in controlled conditions [5].

The neural sequence generating sleep and wake is a complex chain influenced by physiological factors and pathological conditions. Mainly, the sleep/wake cycle regulatory system includes an ascending network extending from the medulla to the forebrain and involving thalamus, basal forebrain, posterior hypothalamus, and brainstem monoaminergic nuclei e.g. noradrenergic neurons of the locus coeruleus (LC) and serotonergic neurons of the dorsal raphe [6]. Dopamine neurons of the substantia nigra and ventral tegmental area, glutamate, hypocretin and the histaminergic tuberomammillary nucleus are all involved innervating through the forebrain the cerebral cortex [7]. The striatum, vital for motor control and cognition, has extensive functional interactions with multiple brain structures e.g. the hippocampus, thalamus and the prefrontal cortex. Interactions between hippocampus, striatum and the cerebral cortex generate sleep-related memory consolidation processes [8]. It is proposed that sleep can reorganize the activity within, as well as the functional interactions between these structures [9]. In fact, several studies describe the modulating effects of sleep deprivation on monoaminergic network $[10,11]$. For instance, sleep is related to the amygdalo-striatal system that is persistent

\footnotetext{
* Corresponding author at: Faculdade de Medicina, Universidade Federal do Ceará, R. Cel Nunes de Melo 1315 - CEP 60.430-270, Fortaleza, Ceará, Brazil.

E-mail addresses: danielethiago@yahoo.com.br (T.M.d.C. Daniele), pfelipe@superig.com.br (P.F.C. de Bruin), emilianovascocelos@yahoo.com.br (E.R.V. Rios), veralice@superig.com.br (V.M.S. de Bruin).

${ }^{1}$ Equal contributions.
} 
throughout evolution and constitutes an essential part of the primordial emotional brain $[3,12]$.

Animal and human experiments have provided evidence for the role of striatum in sleep [13]. In the rotenone experimental model of Parkinson's disease, Rapid Eye Movement (REM) sleep deprivation compromises memory similarly to a nigrostriatal lesion [14]. Durrant et al., testing auditory memory and using polysomnography and magnetic resonance in healthy volunteers, showed that weaker parahippocampal responses and stronger striatal responses occurred after sleep and this was predicted by the amount of slow wave sleep [8]. The latest findings provide further evidence consolidating the relationship of the striatum with sleep [15].

Physical exercise positively modifies mood symptoms and mobility $[16,17]$. Moreover, exercise improves sleep quality [18], memory [19] and influences anti-inflammatory activity [20]. Importantly, a study evaluating the effects of exercise in Parkinson's disease showed that physical activity improved mobility and general signs of the illness [21]. Sleep disturbances are common in neurodegenerative conditions, e.g. Parkinson's disease, influencing motor performance, cognition, mood and therapeutic response [22]. Given that sleep alterations are common in Parkinson's disease and exercise improves disease related symptoms, it is important to investigate, in conditions of sleep deprivation, how exercise affects behavior and the striatum.

The objective of this study is to evaluate the effects of a treadmill exercise protocol on behavior and neurochemical alterations in the striatum in mice submitted to two protocols of sleep deprivation: $6 \mathrm{~h}$ of total sleep deprivation (6h-TSD) and $72 \mathrm{~h}$ of REM sleep deprivation (72h-REMSD).

\section{Material and methods}

\subsection{Animals}

The experimental protocol involved 60 male adult Swiss mice, weighing 25-30 g housed in standard conditions (light-dark cycle: $12 \mathrm{~h} / 12 \mathrm{~h}$, temperature: $23 \pm 1{ }^{\circ} \mathrm{C}$, humidity: $50 \pm 10 \%$ ) with food and water ad libitum. The study followed the ethical principles of animal experimentation established by the Brazilian College of Animal Experimentation (COBEA) and was previously approved by the Ethics Committee in Animal Research (CEPA-67-09).

Animals were grouped (4-5 per cage) Experiment was in accordance with the guidelines established by the Ethical and Practical Principles for the Use of Laboratory Animals [23].

\subsection{Experimental procedures}

Fig. 1 shows an overview of the experiments. Six groups of animals ( $\mathrm{N}=10$ each) were distributed, as described. Group 1 was control kept in their home cages. Group 2 was submitted to 6 h-TSD and group 3 to 72h-REMSD. Group 4 performed up to one-hour of treadmill exercise for 8 weeks. Group 5 included mice that previously exercised (1-h treadmill exercise for 8 weeks) and were right away submitted to 6hTSD; group 6 included mice that previously exercised (1-h treadmill exercise for 8 weeks) and were immediately REM sleep deprived for $72 \mathrm{~h}$ (72h-REMSD).

Time to initiation of sleep deprivation was around Zeitberg Time (ZT) 6 and exercise was also conducted at daytime, under conditions of darkness, from 11:00 AM to 01:00 PM (ZT 6-7). Behavioral tests, firstly, the Elevated Plus Maze (5 min) and secondly, Tail Suspension (6 min), were performed, immediately at the end of exercise or sleep deprivation period. Behavioral tests were scored manually by a blinded examiner.

Animals were decapitated immediately after behavioral tests (11 $\mathrm{min}$ ) and the striatum (dorsal and ventral) was dissected and frozen for analysis.
Group 1

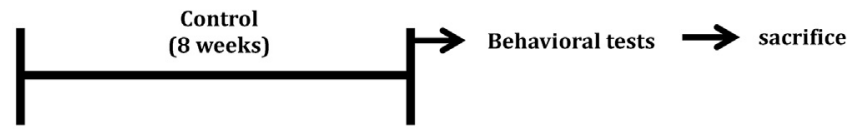

Group 2 and 3

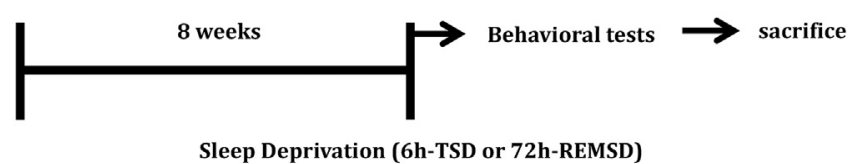

Group 4

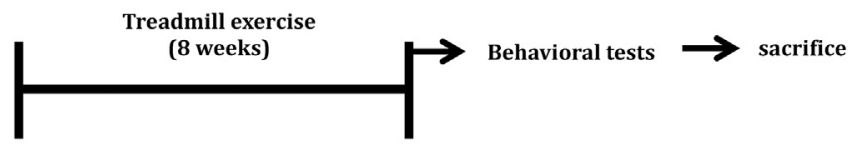

Group 5

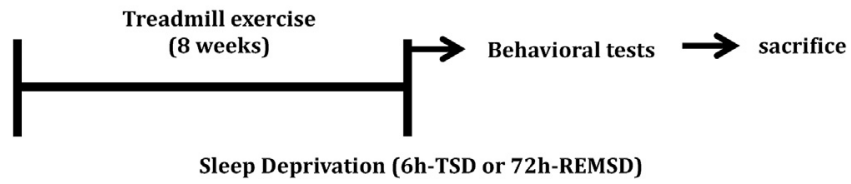

Fig. 1. Overview of experiments. Group 1 was control kept in their home cages. Groups 2 and 3 were subjected to sleep deprivation (6h-TSD or 72h-REMSD). Group 4 performed up to one-hour of treadmill exercise only. Groups 5 and 6 were previously exercised and then subjected to sleep deprivation (6h-TSD or 72h-REMSD). Time to initiation of sleep deprivation was Zeitberg Time (ZT) 6 and exercise was conducted at daytime from 11:00 AM to 01:00 PM (ZT 6-7).

\subsection{Sleep deprivation}

\subsubsection{Total sleep deprivation (6h-TSD)}

Mice were sleep deprived during $6 \mathrm{~h}$ of the light phase (ZT6 to ZT12) by gentle handling (cage tapping and delicate touching). Gentle handling method ( $6 \mathrm{~h})$ is described by Fenzl et al. [24] and Franken et al. [25]. Food and water were available ad libitum. Mice were acclimatized for similar conditions and housed for at least 2 weeks prior to experimental use.

\subsubsection{REM sleep deprivation (72h-REMSD)}

Sleep deprivation (72h-REMSD) was initiated at ZT6 using the multiple platform method adapted for mice [26]. Groups of 5 mice were placed on platforms in tanks $(32 \mathrm{~cm} \times 42 \mathrm{~cm} \times 18 \mathrm{~cm})$ for $72 \mathrm{~h}$. Each tank contained 14 platforms ( $3 \mathrm{~cm}$ in diameter) surrounded by water up to one $\mathrm{cm}$ beneath the surface of the platforms. Food and water were available through a grid placed on top of the water tank through all experiments. In this model, animals can move inside the tank by jumping from one platform to another and restriction of movement or social isolation are not imposed. The "flower pot" method is the best method to selectively deprive animals of rapid eye movement (REM) sleep for one or multiple days with only intermittent monitoring by the researcher [27]. When the characteristic muscle atonia occurs, the animal contact the water surrounding the platform and immediate awakening occur.

\subsection{Exercise protocol}

All groups were habituated on an eight-channel motor-drive treadmill (Model Insight ${ }^{\circledR}$-Equipment, Research and Education-Co., Brazil). Treadmill exercise was also conducted during daytime, under condi- 
A

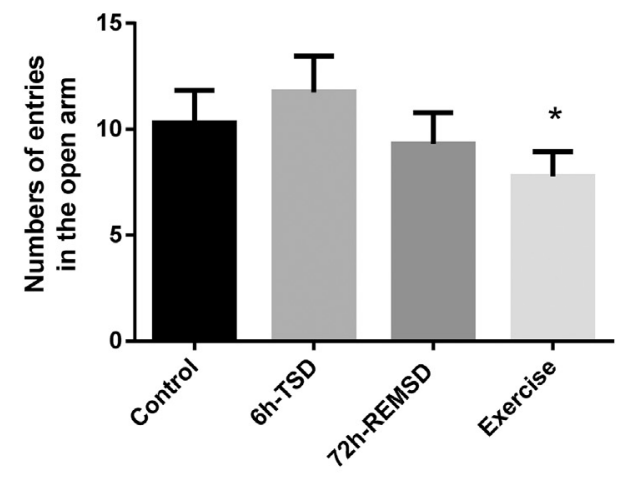

c

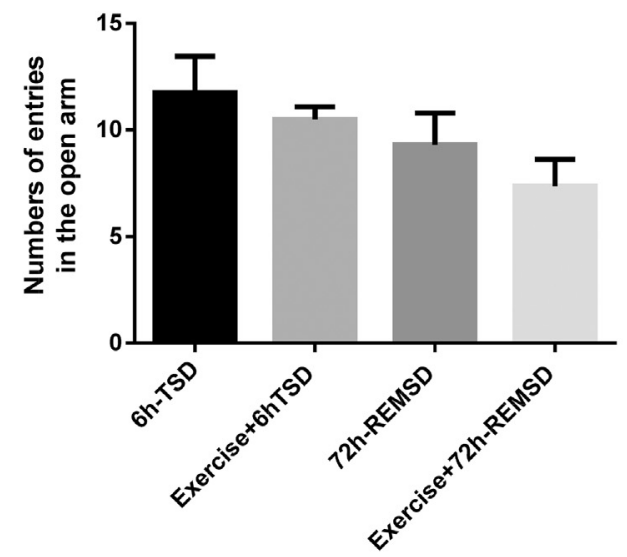

B

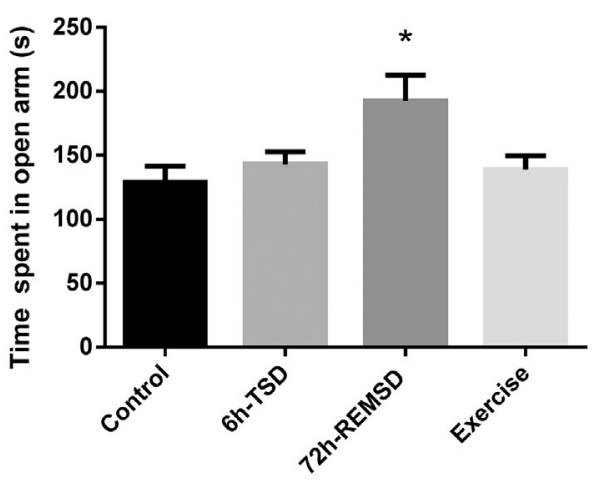

D

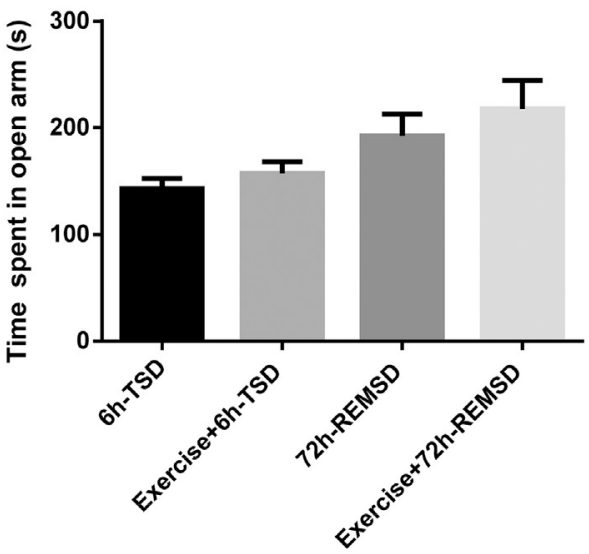

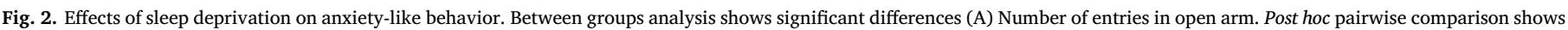

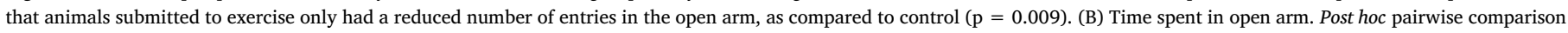

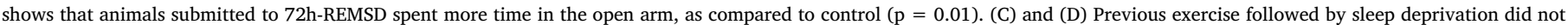
significantly modify number of entries and time spent in the open arm (ANOVA followed by using Tukey's HSD test). *p $<0.05$, **p $<0.001$.

tions of darkness, from 11:00 AM to 01:00 PM (ZT 6-7).

One week of treadmill familiarization ( $5 \mathrm{~min} /$ day, with time-out on weekends) was done to eliminate novel effects. Time and speed were adjusted weekly. Exercises were done 5 days per week, with a progressive increase from $10 \mathrm{~min}$ up to $60 \mathrm{~min}$ for 8 weeks, and a $0^{\circ}$ inclination. Exercise was always at the same time of the day (ZT 6-7). Before running, mice were firstly acclimatized by placing them on an unmoving treadmill for $10 \mathrm{~min}$, then at $5 \mathrm{~m} / \mathrm{min}$ for $10 \mathrm{~min}$, and at $10 \mathrm{~m} / \mathrm{min}$ for $10 \mathrm{~min}$ on succeeding days for 5 days before testing; the speed was increased until $13 \mathrm{~m} / \mathrm{min}$. The control group was left in the treadmill, without running, for the same amount of time as the exercise group [28].

To minimize stress associated with treadmill exercise, only gentle tail touching was used to induce mice to run, and no electric or voice stimulant was used. None of the animals were visibly hurt or died during exercise sessions.

\subsection{Behavioral tests}

\subsubsection{Elevated plus maze test}

The elevated plus maze consisted of two open arms $(28.5 \times 7 \mathrm{~cm})$ and two closed arms $(28.5 \times 7 \times 14 \mathrm{~cm})$ arranged perpendicularly [29]. The maze was elevated $50 \mathrm{~cm}$ above the floor. Each mouse was placed in the center of the apparatus and the number of entries and time spent in the open arms were recorded for $5 \mathrm{~min}$.

\subsubsection{Tail suspension test}

Mice, both acoustically and visually isolated, were suspended $50 \mathrm{~cm}$ above the floor by the tip of the tail $(1 \mathrm{~cm})$. The total test procedure of mouse immobility time was counted during a test period of $6 \mathrm{~min}$. Mice were considered immobile only when they hung passively and completely motionless [30].

\subsection{Brain tissue analysis - Striatal monoamine levels}

Striatum was collected and used to prepare 10\% homogenates for the measurement of norepinephrine (NE), serotonin (5-HT), 5-hydroxyindoleacetic acid (5-HIAA) levels and 5-HT turnover (5-HIAA/5-HT). Homogenates were sonicated in $0.1 \mathrm{M} \mathrm{HClO} 4$, for $30 \mathrm{~s}$, centrifuged at $4{ }^{\circ} \mathrm{C}$ for $15 \mathrm{~min}$ at $15,000 \mathrm{rpm}$, and the supernatant was filtered $(0.2 \mu \mathrm{m}$, Millipore). A $20 \mu \mathrm{l}$ sample was then injected into a highperformance liquid chromatography (HPLC) system (Shimatzu Corp., Japan). Was used a column, as fixed phase, Shimadzu CLC-ODS (M) for analysis. The mobile phase was $0.163 \mathrm{M}$ citric acid, $\mathrm{pH}$ 3.0, containing $0.02 \mathrm{mM}$ EDTA, with $0.69 \mathrm{mM}$ sodium octanesulfonic acid (SOS), as ion pairing reagent, $4 \% \mathrm{v} / \mathrm{v}$ acetonitrile and $1.7 \% \mathrm{v} / \mathrm{v}$ tetrahydrofuran. $\mathrm{NE}$, serotonin and 5-HIAA standards samples were applied to HPLC with electrochemical detection (amperometric detector-Model L-ECD-6A; Shimadzu Corp., Japan), by oxidation of a glassy carbon electrode at $0.85 \mathrm{~V}$ relative to the $\mathrm{Ag}-\mathrm{AgCl}$ reference electrode. The amount of monoamines was determined by comparison with standards injected into the HPLC system on the day of experiment, and concentrations 
were expressed as micrograms $\mu / g$ of tissue.

\subsection{Statistical analyses}

Descriptive statistics are presented as mean ( \pm standard error). All data were tested for normal distribution and equal variance. Analysis of variance (ANOVA) followed by post hoc Tukey's test was employed to compare results between multiple groups and pairwise comparisons. Statistical analysis was carried out using SPSS for Windows, version 21.0. Statistical significance was set at $\mathrm{p}<0.05$.

\section{Results}

\subsection{Elevated plus maze test (Fig. 2)}

3.1.1. Compared groups: control, $6 \mathrm{~h}$ total sleep deprivation (6h-TSD), $72 \mathrm{~h}$ REM sleep deprivation (72h-REMSD) and exercise only

Number of entries in the open arm (ANOVA, $F=6.02, p=0.002$ ) and time spent in the open arms (ANOVA, $\mathrm{F}=4.10, \mathrm{p}=0.01$ ) were significantly different between groups. Post hoc pairwise comparison using Tukey's HSD test showed that animals submitted to exercise only had a reduced number of entries in the open arm ( $\mathrm{p}=0.009$; Fig. 2A). Post hoc pairwise comparison using Tukey's HSD test showed that animals submitted to 72h-REMSD spent more time in the open arm $(\mathrm{p}=0.01 ;$ Fig. 2B)

3.1.2. Compared groups: $6 h-T S D, 72 h-R E M S D$ and previous exercise immediately followed by $6 h-T S D$ and $72 h-R E M S D$

Number of entries in open arm (ANOVA, $F=3.31, p=0.03$ ) and time spent in the open arms (ANOVA, $\mathrm{F}=3.10, \mathrm{p}=0.04$ ) were significantly different between groups. However, post hoc pairwise comparison using Tukey's HSD test did not show significant differences. In short, previous exercised followed by 72h-REMSD or 6h-TSD did not modify time or number of entries in the open arm (Fig. 2C and D).

\subsection{Total immobilization time (Fig. 3)}

3.2.1. Compared groups: control, $6 h-T S D, 72 h-R E M S D$ and exercise only

Between groups analysis showed significant differences in the tail suspension test (ANOVA, $\mathrm{F}=469.6, \mathrm{p}<0.005$ ). Post hoc pairwise comparison using Tukey's HSD test showed that animals subjected to 6h-TSD and 72h-REMSD had increased time of immobilization ( $<<0.005$; Fig. 3A). Exercise only also increased the time of immobilization $(\mathrm{p}=0.001$; Fig. $3 \mathrm{~A})$.

3.2.2. Compared groups: 6h-TSD, 72h-REMSD and previous exercise followed by $6 h-T S D$ and $72 h-R E M S D$

Between groups analysis showed significant differences in the tail suspension test (ANOVA, $\mathrm{F}=302.0, \mathrm{p}<0.005$ ). Post hoc pairwise comparison using Tukey's HSD test showed that previous exercise reduced the time of immobilization after $6 \mathrm{~h}$-TSD $(\mathrm{p}=0.002)$ and after 72h-REMSD ( $\mathrm{p}=0.01$; Fig. 3B).

\subsection{Brain monoamines}

\subsubsection{Norepinephrine levels in the striatum (Fig. 4)}

3.3.1.1. Compared groups: control, 6h-TSD, 72h-REMSD and exercise only. Between groups analysis showed significant differences in NE levels in the striatum (ANOVA, $\mathrm{F}=39.01, \mathrm{p}<0.005$ ). Post hoc pairwise comparison using Tukey's HSD test showed that mice submitted to 6h-TSD ( $\mathrm{p}<0.005$; Fig. 4A) and to 72h-REMSD $(\mathrm{p}=0.001$; Fig. 4A) had higher levels of NE. Exercise only also increased NE levels ( $p=0.04$; Fig. 4A). followed by $6 h-T S D$ and $72 h-R E M S D$. Between groups analysis showed
A

Total imobilization

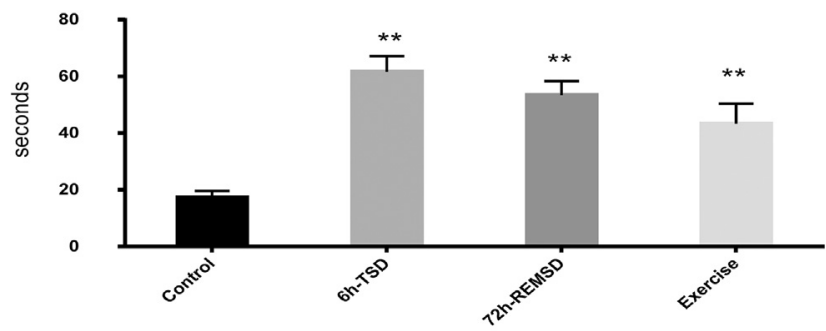

B

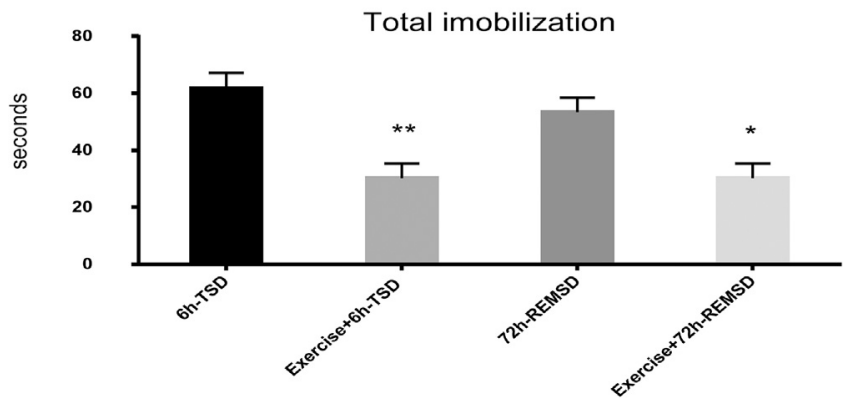

Fig. 3. Effect of treadmill exercise on depressive symptoms (tail suspension) in sleepdeprived mice. Between groups analysis shows significant differences in the tail suspension test (A) Total time spent immobile. Post hoc pairwise comparison shows that 6h-TSD ( $\mathrm{p}<0.005$ ), 72h-REMSD ( $\mathrm{p}<0.005$ ) and exercise only increased time of immobilization $(\mathrm{p}=0.001)$. (B) Total time spent immobile. Between groups analysis shows significant differences in the tail suspension test $(\mathrm{p}<0.005)$. Post hoc pairwise comparison shows that previous exercise reduces the time of immobilization after 6h-TSD ( $\mathrm{p}=0.002)$ and after 72h-REMSD $(\mathrm{p}=0.01)$ (ANOVA followed by Tukey's HSD test) *p $<0.05, * * \mathrm{p}<0.001$.

significant differences in NE levels in the striatum (ANOVA, $\mathrm{F}=41.02$, $\mathrm{p}<0.005$ ). Post hoc pairwise comparison using Tukey's HSD test showed that mice subjected to previous exercise followed by 6h-TSD ( $<<0.005$; Fig. 4B) or by 72h-REMSD ( $\mathrm{p}=0.001$; Fig. 4B) had reduced levels of $\mathrm{NE}$ in the striatum as compared to sleep deprivation only.

3.3.2. Serotonin, 5-HIAA levels and serotonin turnover in the striatum (Fig. 5)

3.3.2.1. Compared groups: control, 6h-TSD, 72h-REMSD and exercise only. Between groups analysis showed significant differences in serotonin levels in the striatum (ANOVA, $\mathrm{F}=14.28, \mathrm{p}<0.005$ ). Post hoc pairwise comparison using Tukey's HSD test showed that animals submitted to exercise only had higher serotonin levels ( $\mathrm{p}<0.005$, Fig. 5A). Sleep deprivation, both models 6h-TSD and 72h-REMSD, did not modify serotonin levels.

Between groups analysis showed significant differences in 5-HIAA levels in the striatum (ANOVA, $\mathrm{F}=41.38, \mathrm{p}<0.005$ ). Post hoc pairwise comparison using Tukey's HSD test showed that mice subjected to $6 \mathrm{~h}$-TSD had higher levels of 5-HIAA ( $\mathrm{p}=0.004$; Fig. 5B). Exercise only also increased 5-HIAA levels ( $\mathrm{p}=0.04$; Fig. 5B).

Between groups analysis showed significant differences in serotonin turnover (ANOVA, $\mathrm{F}=52.23, \mathrm{p}<0.005$ ). Post hoc pairwise comparison using Tukey's HSD test showed that mice subjected to 6h-TSD had higher serotonin turnover ( $<<0.005$; Fig. $5 \mathrm{C}$ ). Exercise only also showed increased serotonin turnover ( $p=0.006$; Fig. $5 C$ ).

3.3.2.2. Compared groups: $6 h-T S D, 72 h-R E M S D$ and previous exercise followed by $6 h-T S D$ and 72h-REMSD. Regarding serotonin levels, 


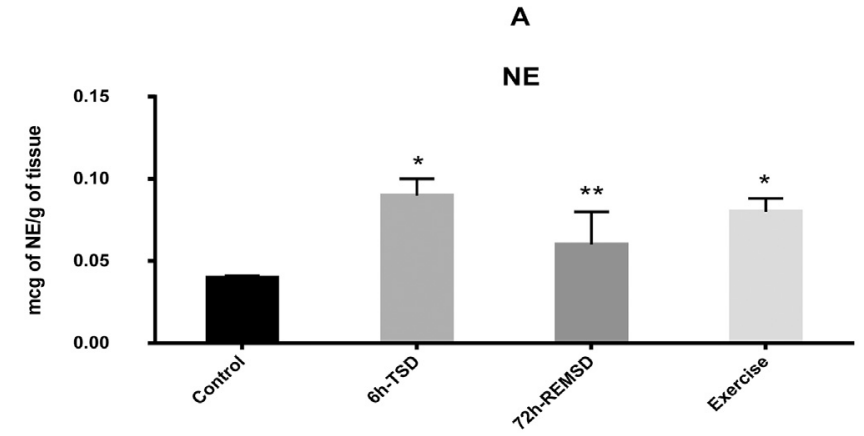

B

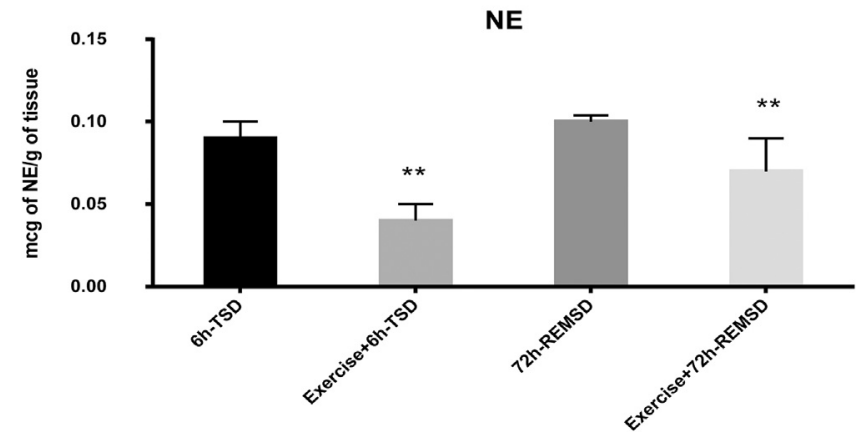

Fig. 4. Alterations in norepinephrine (NE) levels in the striatum after sleep deprivation, exercise and combined actions. Between groups analysis shows significant differences in NE levels (A) Post hoc pairwise comparison shows that 6h-TSD ( $\mathrm{p}<0.005)$, 72h-REMSD $(\mathrm{p}=0.001)$ and exercise $(\mathrm{p}=0.04)$ increased NE levels. (B) Post hoc pairwise comparison shows that previous exercise followed by 6h-TSD ( $\mathrm{p}<0.005)$ and 72h-REMSD $(\mathrm{p}=0.001)$ reduced NE levels (ANOVA followed by Tukey's HSD test). ${ }^{*} \mathrm{p}<0.05$, $* * \mathrm{p}<0.001$. between groups analysis showed significant differences in the striatum (ANOVA, $\mathrm{F}=4.75, \mathrm{p}=0.01$ ). Post hoc pairwise comparison using Tukey's HSD test showed that animals submitted to exercise followed by 72h-REMSD increased levels of serotonin ( $\mathrm{p}=0.04$; Fig. 5D).

Regarding 5-HIAA levels, between groups analysis showed significant differences in the striatum (ANOVA, $\mathrm{F}=5.13, \mathrm{p}=0.01$ ). Post hoc pairwise comparison using Tukey's HSD test did not show significant differences (Fig. 5E).

Regarding serotonin turnover, between groups analysis showed significant differences in the striatum (ANOVA, $F=33.50$, $\mathrm{p}<0.005)$. Post hoc pairwise comparison using Tukey's HSD test showed that animals submitted to exercise followed by 6h-TSD or 72hREMSD had reduced levels of serotonin turnover (5-HIAA/5-HT) ( $\mathrm{p}<0.005$ and $\mathrm{p}=0.04$, respectively; Fig. 5F).

\section{Discussion}

This study shows that exercise reduces depressive behavior, levels of $\mathrm{NE}$ and serotonin turnover measures in association with sleep deprivation in the striatum. The same results were consistently found in both experimental models of sleep deprivation, 6h-TSD and 72h-REMSD, strengthening the validity of the findings. In short, exercise changed behavior and neurochemistry in association with sleep deprivation. We will subsequently discuss conflicting evidence regarding depressive and anxiety behavior in animal models of sleep deprivation and the significance of the striatal monoamine results.

Previously, reports on the effects of acute sleep deprivation on anxiety are controversial: some experiments point to anxiogenesis and others to anxiolysis [31]. The lack of change in anxiety behavior after sleep deprivation, presently found in this study, is in agreement with other report involving sleep restriction in rats [32]. Furthermore, some argue that the increased number of entries and/or time spent in open arm found in this model is justified by mania rather than to an anxiolytic effect [33].

In agreement with previous reports, our results show that sleep deprivation induces depressive behavior [34]. Clinical evidence shows
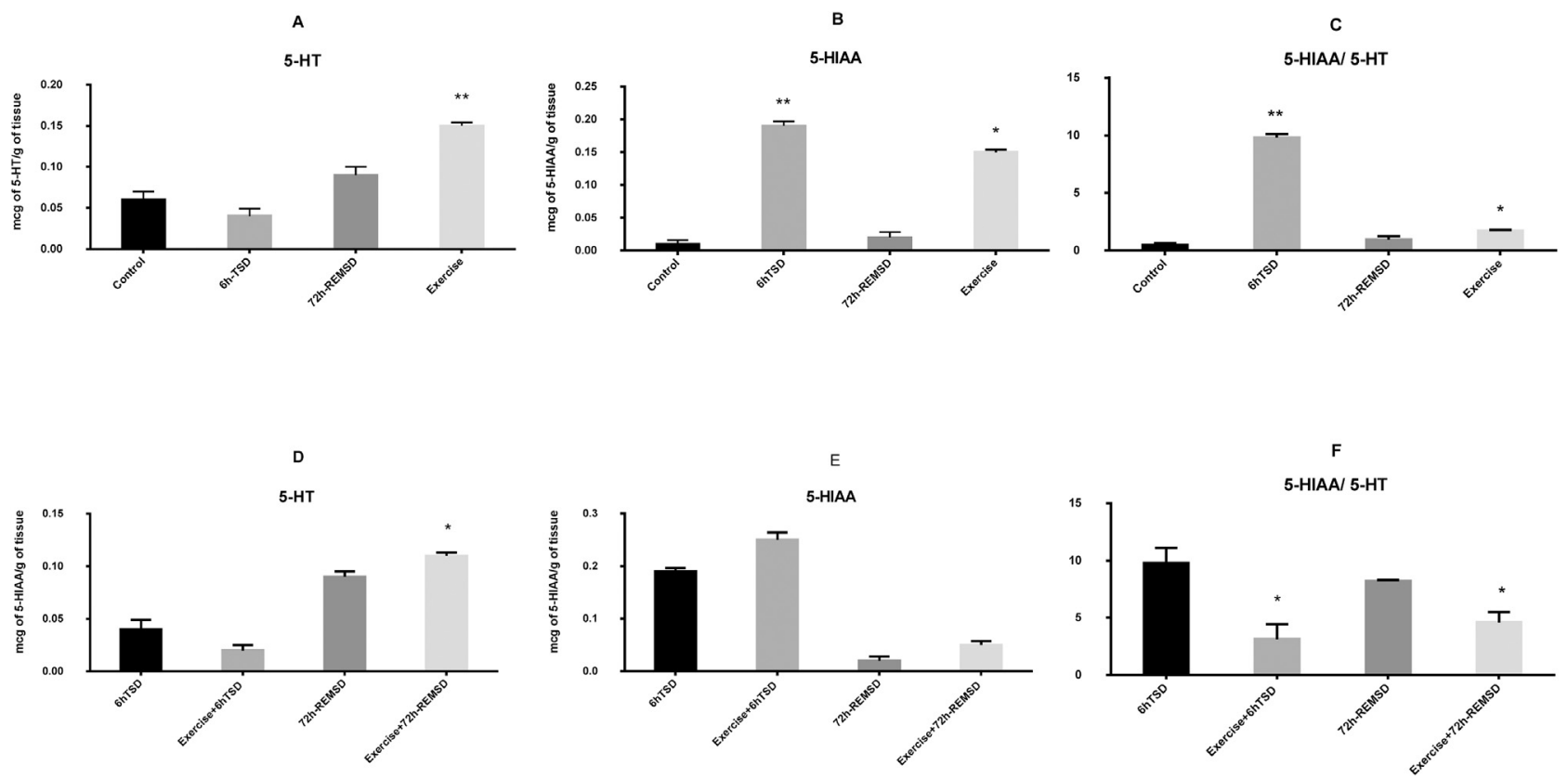

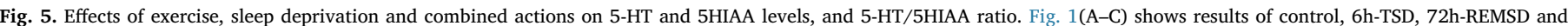

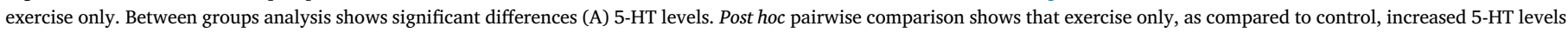

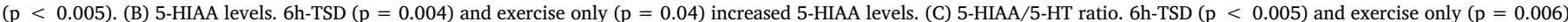

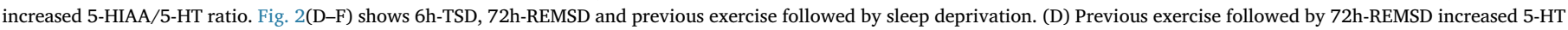

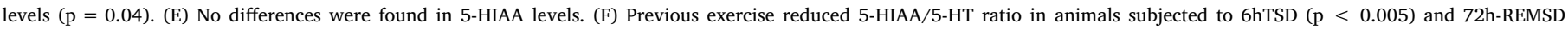
$(\mathrm{p}=0.04) .{ }^{*} \mathrm{p}<0.05,{ }^{* *} \mathrm{p}<0.001$. 
that the relationship between sleep deprivation and depression is complex: sleep deprivation aggravates depression [35]; and conversely, it is used as a chronotherapeutic method of treatment [36]. The amelioration of depressive symptoms after sleep deprivation is transient and unsustained [37]. The mechanistic basis for these, somehow, contradictory findings need more clarification.

Effects of exercise on behavior have also been a focus of controversy: exercise is associated with both, increased [38] and reduced anxiety [39]. In this work, exercise elicited a reduction in the number of entries in open arm, an indicative of elevated anxiety. These data are in accordance with other rodent studies $[40,41]$. In contradiction, other researchers report that treadmill training reduces anxiety-like behaviors [42] Schoenfeld [43]. Furthermore, our results showed that previous exercise reduce depressive behavior. These findings are directly in agreement with clinical [44] and animal experiment [45] evidence reaffirming that exercise improves depression.

In the present study, mice submitted to exercise showed higher levels of serotonin turnover. This is in agreement with a previous report showing an improvement of depressive symptoms and high striatal serotonin turnover after aerobic exercise $[17,46]$. Clinical study shows that unmedicated patients with major depression show higher serotonin turnover levels [47]. Additionally, the use of selective serotonin reuptake inhibitors and improvement of symptoms is associated with a reduction of serotonin turnover [48]. Judging from these findings, a reduction of serotonin turnover is directly associated with the improvement of depression. Our results show that in both models of sleep deprivation, 6h-TSD and 72h-REMSD, previous exercise reduced depressive symptoms and striatal serotonin turnover. Brain serotonin turnover is a strong indicator of rates at which serotonin and its metabolites enter and leave the brain [49].

Importantly and in agreement with our findings, former work confirms that REM sleep deprivation increases NE level in the brain [50,51]. In fact, high NE levels occurred in both models of sleep restriction: the 6h-TSD, a milder form, and the 72h-REMSD, of a more severe nature. It is known that high NE levels stimulate neuronal $\mathrm{Na}^{+}$$\mathrm{K}^{+}$-ATPase, a fundamental factor for maintaining neuronal excitability [52]. Considering that REM sleep deprivation increases cerebral NE levels, Mallick e Singh [53] postulated that REM sleep possibly serves a housekeeping function in the brain. Furthermore, they propose that subjects suffering from REM sleep loss may be effectively treated by reducing either $\mathrm{NE}$ level or $\mathrm{Na}^{+}-\mathrm{K}^{+}$-ATPase activity in the brain. In this study, in both models, previous exercise reduced the high levels of NE in the striatum. This effect was more intense in the 6h-TSD model and less potent in the 72h-REMSD. This could be explained by the fact that 72h-REMSD affects more aggressively the brain.

At present, a clear understanding on how treadmill exercise reduces $\mathrm{NE}$ in the striatum of sleep deprived animals is not available. A burn out effect on the striatum caused by exercise followed by sleep deprivation cannot be excluded. The question whether this could be a protective effect against the possible toxic injury induced by very high levels of $\mathrm{NE}$ remains to be answered. Moreover, how could this be linked to improvement of depressive behavior in this model is also to be clarified.

Limitations to this study must be acknowledged. Regrettably, nearly all experimental models of sleep deprivation involve associated stress. In the present study, we performed two models of sleep deprivation: $6 \mathrm{~h}$ using gentle handling and $72 \mathrm{~h}$ using the flower pot method. Sleep analysis should have been performed. Of value, this research shows how these two models of sleep deprivation differentially affects behavioral and brain alterations.

Additionally, conditioned exercise also inflicts a certain degree of stress. Less stressful forms of exercise e.g. wheel running do not guarantee the same amount of exercise for all. Studies on the effects of exercise have included different protocols varying in intensity and duration. Studies using one day [54], 5 days [55] and 26 weeks [56] were described. This variation by itself hampers comparisons. In this work, eight weeks of exercise was considered a good average con- ditioning protocol.

In this experiment, we focused only in the striatum. Other brain areas were unfortunately not included. In fact, previously, structures with important circuitry related to sleep, learning, memory and motor function e.g. thalamus, striatum and the substantia nigra were rarely investigated [57-59]. Very few studies described the effects of exercise in connection with sleep deprivation in mice, particularly brain tissue changes $[39,60,61]$. This study provides evidence regarding alterations of striatal monoamines in connection with sleep deprivation and exercise.

The interpretation of neurochemical results must take into consideration that determinations were obtained from brain tissue homogenates. The homogenized tissue determines the amount within the cells as well as extracellular space. Therefore, the use of microdialysis would be more appropriate. Furthermore, the homogenization process will mix enzymes that can degrade the neurotransmitters: neurotransmitters have short lives as they can break down extremely quickly [6]. More complicated is the fact that their widespread distribution in many networks adds to the difficulties in analyzing their functions and interactions. Interestingly, a previous study evaluating monoamines in the cerebral tissue showed that the NE and serotonin contents were relatively homogenous. In addition, the regulation of these neuromodulatory systems is probably achieved mainly at the terminals. Importantly, each of these systems contributes to the regulation of the other [49]. Hence, information regarding the content of $\mathrm{NE}$ and serotonin in the brain is useful and the striatum as a midline structure with multiple connections has a crucial role in motricity and behavior.

Importantly, the study of other biomolecules may contribute to an understanding of the present findings. For instance, increased resilience associated with exercise related to the presence of galanin, a neuropeptide produced in the LC. This needs further investigation [62,63]. Running amplifies galanin expression in noradrenergic LC. It also suppresses stress-induced activity of the LC and norepinephrine output in LC-target regions [62]. All this evidence suggests that chronic exercise may inhibit noradrenergic LC-neurons [64].

In conclusion, this study using two models of sleep deprivation in mice, 6h-TSD and 72h-REM SD, showed that exercise reduced depressive behavior, the levels of $\mathrm{NE}$ and of serotonin turnover in the striatum. The present findings add to the understanding of behavior and brain alterations in connection with sleep and exercise.

\section{Relevant conflicts of interests/financial disclosures}

The authors declare no conflicts of interest.

\section{Funding agency}

The work that led up to this study was supported by Fundação Cearense de Desenvolvimento Tecnológico (FUNCAP) and Conselho Nacional de Desenvolvimento Científico (CNPq).

\section{References}

[1] M. Engle-Friedman, The effects of sleep loss on capacity and effort, Sleep Sci. (Sao Paulo, Brazil) 7 (2014) 213-224.

[2] J.D. Sweatt, K.E. Hawkins, The molecular neurobiology of the sleep-deprived, fuzzy brain, Sci. Signal. 9 (2016) fs7.

[3] R. Gruber, J. Cassoff, The interplay between sleep and emotion regulation: conceptual framework empirical evidence and future directions, Curr. Psychiatry Rep. 16 (2014) 500.

[4] L. Xie, H. Kang, O. Xu, M.J. Chen, Y. Liao, M. Thiyagarajan, et al., Sleep drives metabolite clearance from the adult brain, Science (New York, NY) 342 (2013) 373-377.

[5] L.A. Toth, P. Bhargava, Animal models of sleep disorders, Comp. Med. 63 (2013) 91-104.

[6] A. Lajtha, E.S. Vizi, Handbook of Neurochemistry and Molecular Neurobiology: Neurotransmitter Systems, 3rd ed., Spring, 2008.

[7] I.T. French, K.A. Muthusamy, A review of sleep and its disorders in patients with Parkinson's disease in relation to various brain structures, Front. Aging Neurosci. 8 
(2016) 114.

[8] S.J. Durrant, S.A. Cairney, P.A. Lewis, Overnight consolidation aids the transfer of statistical knowledge from the medial temporal lobe to the striatum, Cereb. Cortex 23 (2013) 2467-2478.

[9] G. Albouy, B.R. King, P. Maquet, J. Doyon, Hippocampus and striatum: dynamics and interaction during acquisition and sleep-related motor sequence memory consolidation, Hippocampus 23 (2013) 985-1004.

[10] K.S. Murnane, M.L. Andersen, K.C. Rice, L.L. Howell, Selective serotonin 2A receptor antagonism attenuates the effects of amphetamine on arousal and dopamine overflow in non-human primates, J. Sleep Res. 22 (2013) 581-588.

[11] M.B. Proenca, P.A. Dombrowski, C. Da Cunha, L. Fischer, A.C. Ferraz, M.M. Lima, Dopaminergic D2 receptor is a key player in the substantia nigra pars compacta neuronal activation mediated by REM sleep deprivation, Neuropharmacology 76 (Pt. A) (2014) 118-126.

[12] W.D. Killgore, Self-reported sleep correlates with prefrontal-amygdala functional connectivity and emotional functioning, Sleep 36 (2013) 1597-1608.

[13] A.S. Elliott, J.D. Huber, J.P. O'Callaghan, C.L. Rosen, D.B. Miller, A review of sleep deprivation studies evaluating the brain transcriptome, Springerplus 3 (2014) 728.

[14] A.C. Dos Santos, M.A. Castro, E.A. Jose, A.M. Delattre, P.A. Dombrowski, C. Da Cunha, et al., REM sleep deprivation generates cognitive and neurochemical disruptions in the intranigral rotenone model of Parkinson's disease, J. Neurosci. Res. 91 (2013) 1508-1516.

[15] A.M. Lima, V.M. de Bruin, E.R. Rios, P.F. de Bruin, Differential effects of paradoxical sleep deprivation on memory and oxidative stress, NaunynSchmiedeberg's Arch. Pharmacol. 387 (2014) 399-406.

[16] E.S.B. Andrade, R.C. Cassilhas, C. Attux, Q. Cordeiro, A.L. Gadelha, B.A. Telles, et al., A 20 -week program of resistance or concurrent exercise improves symptoms of schizophrenia: results of a blind, randomized controlled trial, Rev. Bras. Psiquiatr. 37 (2015) 271-279.

[17] J.H. Roh, I.G. Ko, S.E. Kim, J.M. Lee, E.S. Ji, J.H. Kim, et al., Treadmill exercise ameliorates intracerebral hemorrhage-induced depression in rats, J. Exerc. Rehabil. 12 (2016) 299-307.

[18] D. Lalande, L. Theriault, E. Kalinova, A. Fortin, M. Leone, The effect of exercise on sleep quality and psychological, physiological, and biological correlates in patients with schizophrenia: a pilot study, Schizophr. Res. 171 (2016) 235-236.

[19] H. Saadati, S. Esmaeili-Mahani, K. Esmaeilpour, M. Nazeri, S. Mazhari, V. Sheibani, Exercise improves learning and memory impairments in sleep deprived female rats, Physiol. Behav. 138 (2015) 285-291.

[20] M. Chennaoui, D. Gomez-Merino, C. Drogou, H. Geoffroy, G. Dispersyn, C. Langrume, et al., Effects of exercise on brain and peripheral inflammatory biomarkers induced by total sleep deprivation in rats, J. Inflamm. (Lond.) 12 (2015) 56.

[21] D.M. Corcos, J.A. Robichaud, F.J. David, S.E. Leurgans, D.E. Vaillancourt, C. Poon, et al., A two-year randomized controlled trial of progressive resistance exercise for Parkinson's disease, Mov. Disord. 28 (2013) 1230-1240.

[22] D.G. Tavora, V.M. de Bruin, R. Lopes Gama, E.M. Lopes, I.F. Jorge, P.F. de Bruin, The nature of excessive sleepiness and sudden sleep onset in Parkinsons disease, Sleep Sci. (Sao Paulo, Brazil) 7 (2014) 13-18.

[23] M.L. Anderson, V. D'almeida, M.G. Ko, R. Kawakami, P.J.F. Martins, Princípios éticos e práticos do uso de animais de experimentação, UNIFESP, São Paulo, 2004.

[24] T. Fenzl, C.P. Romanowski, C. Flachskamm, K. Honsberg, E. Boll, A. Hoehne, et al., Fully automated sleep deprivation in mice as a tool in sleep research, J. Neurosci. Methods 166 (2007) 229-235.

[25] P. Franken, I. Tobler, A.A. Borbely, Effects of 12-h sleep deprivation and of 12-h cold exposure on sleep regulation and cortical temperature in the rat, Physiol. Behav. 54 (1993) 885-894.

[26] R.H. Silva, V.C. Abilio, A.L. Takatsu, S.R. Kameda, C. Grassl, A.B. Chehin, et al., Role of hippocampal oxidative stress in memory deficits induced by sleep deprivation in mice, Neuropharmacology 46 (2004) 895-903.

[27] R. Havekes, C.G. Vecsey, T. Abel, The impact of sleep deprivation on neuronal and glial signaling pathways important for memory and synaptic plasticity, Cell. Signal. 24 (2012) 1251-1260.

[28] J.C. Ferreira, N.P. Rolim, J.B. Bartholomeu, C.A. Gobatto, E. Kokubun, P.C. Brum, Maximal lactate steady state in running mice: effect of exercise training, Clin. Exp. Pharmacol. Physiol. 34 (2007) 760-765.

[29] R.G. Lister, The use of a plus-maze to measure anxiety in the mouse, Psychopharmacology (Berl.) 92 (1987) 180-185.

[30] J.F. Cryan, C. Mombereau, A. Vassout, The tail suspension test as a model for assessing antidepressant activity: review of pharmacological and genetic studies in mice, Neurosci. Biobehav. Rev. 29 (2005) 571-625.

[31] G.N. Pires, S. Tufik, M.L. Andersen, Sleep deprivation and anxiety in humans and rodents-translational considerations and hypotheses, Behav. Neurosci. 129 (2015) 621-633.

[32] A. Novati, H.J. Hulshof, J.M. Koolhaas, P.J. Lucassen, P. Meerlo, Chronic sleep restriction causes a decrease in hippocampal volume in adolescent rats, which is not explained by changes in glucocorticoid levels or neurogenesis, Neuroscience 190 (2011) 145-155.

[33] G.S. Kirshenbaum, S.J. Clapcote, S. Duffy, C.R. Burgess, J. Petersen, K.J. Jarowek, et al., Mania-like behavior induced by genetic dysfunction of the neuron-specific $\mathrm{Na}$ ,+ K +-ATPase alpha3 sodium pump, Proc. Natl. Acad. Sci. U. S. A. 108 (2011) 18144-18149.

[34] R. Tadavarty, P.S. Rajput, J.M. Wong, U. Kumar, B.R. Sastry, Sleep-deprivation induces changes in GABA(B) and mGlu receptor expression and has consequences for synaptic long-term depression, PLoS One 6 (2011) e24933.

[35] R.E. Roberts, H.T. Duong, The prospective association between sleep deprivation and depression among adolescents, Sleep 37 (2014) 239-244.

[36] H. Giedke, F. Schwarzler, Therapeutic use of sleep deprivation in depression, Sleep Med. Rev. 6 (2002) 361-377.

[37] E. Dopierala, J. Rybakowski, Sleep deprivation as a method of chronotherapy in the treatment of depression, Psychiatr. Pol. 49 (2015) 423-433.

[38] M. Svensson, P. Rosvall, A. Boza-Serrano, E. Andersson, J. Lexell, T. Deierborg, Forced treadmill exercise can induce stress and increase neuronal damage in a mouse model of global cerebral ischemia, Neurobiol. Stress 5 (2016) 8-18.

[39] M.R. Zielinski, J.M. Davis, J.R. Fadel, S.D. Youngstedt, Influence of chronic moderate sleep restriction and exercise training on anxiety, spatial memory, and associated neurobiological measures in mice, Behav. Brain Res. 250 (2013) 74-80.

[40] T.P. Gavin, P.D. Wagner, Effect of short-term exercise training on angiogenic growth factor gene responses in rats, J. Appl. Physiol. (Bethesda, Md: 1985) 90 (2001) 1219-1226.

[41] T. Gustafsson, W.E. Kraus, Exercise-induced angiogenesis-related growth and transcription factors in skeletal muscle, and their modification in muscle pathology, Front. Biosci. 6 (2001) D75-D89.

[42] L.J. Fulk, H.S. Stock, A. Lynn, J. Marshall, M.A. Wilson, G.A. Hand, Chronic physical exercise reduces anxiety-like behavior in rats, Int. J. Sports Med. 25 (2004) 78-82.

[43] T.J. Schoenfeld, H.C. McCausland, A.N. Sonti, H.A. Cameron, Anxiolytic actions of exercise in absence of new neurons, Hippocampus 26 (2016) 1373-1378.

[44] J. Mota-Pereira, J. Silverio, S. Carvalho, J.C. Ribeiro, D. Fonte, J. Ramos, Moderate exercise improves depression parameters in treatment-resistant patients with major depressive disorder, J. Psychiatr. Res. 45 (2011) 1005-1011.

[45] I. Ensari, R.W. Motl, L.A. Pilutti, Exercise training improves depressive symptoms in people with multiple sclerosis: results of a meta-analysis, J. Psychosom. Res. 76 (2014) 465-471.

[46] T. Matsui, S. Soya, K. Kawanaka, H. Soya, Brain glycogen decreases during intense exercise without hypoglycemia: the possible involvement of serotonin, Neurochem. Res. 40 (2015) 1333-1340.

[47] D.A. Barton, M.D. Esler, T. Dawood, E.A. Lambert, D. Haikerwal, C. Brenchley, et al., Elevated brain serotonin turnover in patients with depression: effect of genotype and therapy, Arch. Gen. Psychiatry 65 (2008) 38-46.

[48] C. Keating, T. Dawood, D.A. Barton, G.W. Lambert, A.J. Tilbrook, Effects of selective serotonin reuptake inhibitor treatment on plasma oxytocin and cortisol in major depressive disorder, BMC Psychiatry 13 (2013) 124.

[49] A. Fitoussi, F. Dellu-Hagedorn, P. De Deurwaerdere, Monoamines tissue content analysis reveals restricted and site-specific correlations in brain regions involved in cognition, Neuroscience 255 (2013) 233-245.

[50] S. Gulyani, B.N. Mallick, Effect of rapid eye movement sleep deprivation on rat brain Na-K ATPase activity, J. Sleep Res. 2 (1993) 45-50.

[51] G. Das, B.N. Mallick, Noradrenaline acting on alpha1-adrenoceptor mediates REM sleep deprivation-induced increased membrane potential in rat brain synaptosomes, Neurochem. Int. 52 (2008) 734-740.

[52] C. Vaillend, S.E. Mason, M.F. Cuttle, B.E. Alger, Mechanisms of neuronal hyperexcitability caused by partial inhibition of $\mathrm{Na}+-\mathrm{K}+$-ATPases in the rat CA1 hippocampal region, J. Neurophysiol. 88 (2002) 2963-2978.

[53] B.N. Mallick, A. Singh, REM sleep loss increases brain excitability: role of noradrenaline and its mechanism of action, Sleep Med. Rev. 15 (2011) 165-178.

[54] N. Pervaiz, L. Hoffman-Goetz, Immune cell inflammatory cytokine responses differ between central and systemic compartments in response to acute exercise in mice, Exerc. Immunol. Rev. 18 (2012) 142-157.

[55] R.T. Enos, J.M. Davis, J.L. McClellan, E.A. Murphy, Indomethacin in combination with exercise leads to muscle and brain inflammation in mice, J. Interferon Cytokine Res. 33 (2013) 446-451.

[56] C.X. Yi, O. Al-Massadi, E. Donelan, M. Lehti, J. Weber, C. Ress, et al., Exercise protects against high-fat diet-induced hypothalamic inflammation, Physiol. Behav. 106 (2012) 485-490.

[57] M. Tanaka, E. Yamaguchi, M. Takahashi, K. Hashimura, T. Shibata, W. Nakamura, et al., Effects of age-related dopaminergic neuron loss in the substantia nigra on the circadian rhythms of locomotor activity in mice, Neurosci. Res. 74 (2012) 210-215.

[58] Z. Chen, R.D. Wimmer, M.A. Wilson, M.M. Halassa, Thalamic circuit mechanisms link sensory processing in sleep and attention, Front. Neural Circuits 9 (2015) 83

[59] M.M. Lim, J. Xu, D.M. Holtzman, R.H. Mach, Sleep deprivation differentially affects dopamine receptor subtypes in mouse striatum, Neuroreport 22 (2011) 489-493.

[60] K. Van der Borght, R. Havekes, T. Bos, B.J. Eggen, E.A. Van der Zee, Exercise improves memory acquisition and retrieval in the Y-maze task: relationship with hippocampal neurogenesis, Behav. Neurosci. 121 (2007) 324-334.

[61] K. van der Borght, F. Ferrari, K. Klauke, V. Roman, R. Havekes, A. Sgoifo, et al., Hippocampal cell proliferation across the day: increase by running wheel activity, but no effect of sleep and wakefulness, Behav. Brain Res. 167 (2006) 36-41.

[62] N.R. Sciolino, P.V. Holmes, Exercise offers anxiolytic potential: a role for stress and brain noradrenergic-galaninergic mechanisms, Neurosci. Biobehav. Rev. 36 (2012) 1965-1984.

[63] T.W. Lin, Y.M. Kuo, Exercise benefits brain function: the monoamine connection, Brain Sci. 3 (2013) 39-53.

[64] R.A. McDevitt, J.F. Neumaier, Regulation of dorsal raphe nucleus function by serotonin autoreceptors: a behavioral perspective, J. Chem. Neuroanat. 41 (2011) $234-246$. 